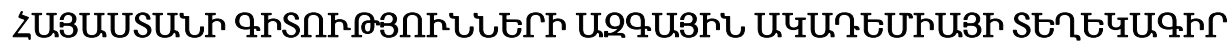 ИЗВЕСТИЯ НАЦИОНАЛЬНОЙ АКАДЕМИИ НАУК АРМЕНИИ
}

Uthumipluu

70, №2, 2017

Механика

\section{AMPLITUDE-PHASE DISTORTION OF THE NORMAL HIGH- FREQUENCY SHEAR WAVES IN HOMOGENEOUS ELASTIC WAVEGUIDE WITH WEAKLY ROUGH SURFACES}

\author{
Avetisyan A.S., Hunanyan A.A.
}

Keywords: instability of normal waves, weakly rough surfaces, delay frequency, internal resonance.

Ключевые слова: неустойчивость нормальной волны, слабо-неоднородные поверхности, полоса задержки частот, внутренний резонанс.

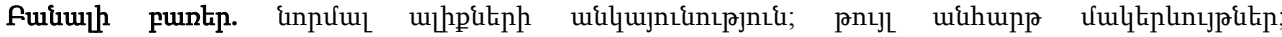

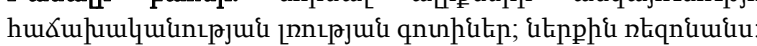

Аветисян А.С., Унанян А.А.

Амплитудно-фазовые искажения высокочастотной нормальной сдвиговой волны в однородном упругом волноводе с слабо-неоднородными поверхностями

Исследуется влияние слабой неоднородности поверхностей упругого слоя-волновода на распространение нормальной сдвиговой волны при механически свободных слабо-неоднородных поверхностях волновода. В приповерхностных зонах волновода виртуально выделяются тонкие прослойки переменной толщины. В выделенных упругих прослойках вводятся функции распределения упругих сдвигов (гипотезы MELS). Ввод гипотез MELS позволяет более подробно исследовать процесс искажения нормальной волны. Оно сделает более удобным исследования волновых процессов в волноводах с усложнёнными свойствами и сложными характерными неоднородностями материала волновода и его поверхностей. Показывается, что в отличие от идеально гладких поверхностей, слабая неоднородность механически свободных поверхностей приводит к искажению распространяющейся нормальной волны. Происходит частичная локализация волновой энергии в приповерхностных прослойках волновода. Появляются частотные зоны умолчания (а также зоны частотного пропускания) вновь формированной волны.

U.lŁunpujuí U.U., Znı\{uiljuir U.U.

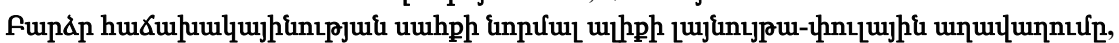

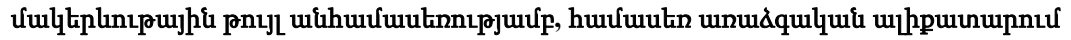

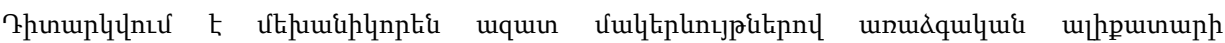

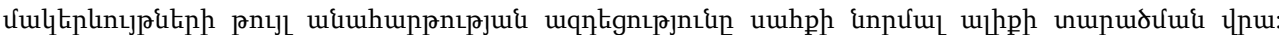

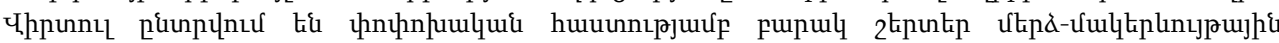

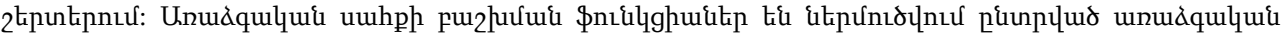

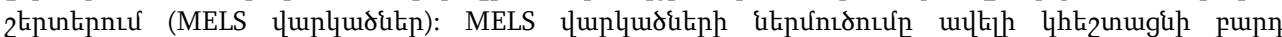

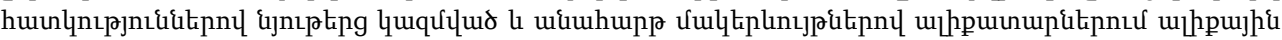

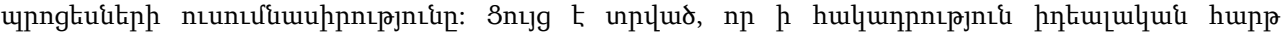

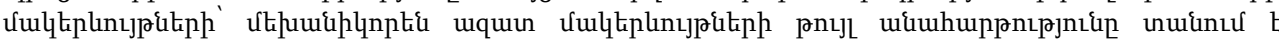

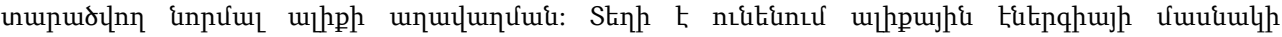

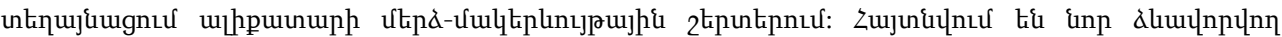

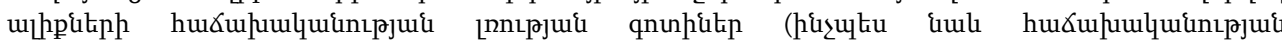

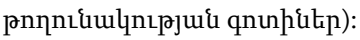

The influence of weak roughness of mechanically free elastic waveguide surfaces on propagation of normal shear wave is investigated. Thin layers of variable thickness are virtually separated in near-surface areas. Distribution functions of elastic shears are introduced in separated elastic layers (hypotheses MELS). Introduction of hypotheses 
MELS will make the study of wave processes in waveguides with complicated properties and sophisticated characteristic roughness of the material of the waveguide and its surfaces more convenient. It is shown that in contrast to perfectly smooth surfaces, weak roughness of the mechanically free surfaces leads to distortion of propagating normal waves. Partial localization of wave energy occurs in near-surface layers of the waveguide. Frequency zones of silence of newly formed waves (as well as zones of frequency bandwidth) appear as well.

\section{Introduction}

The interaction of ultrasound wave with rough surface of waveguides currently is actively investigated from both theoretical and experimental points of view (see e.g. [1-3]). It is related to applications of elastic wave phenomena in modern technology: telecommunications (signal processing), medicine (ultrasound measurement), metallurgy (nondestructive control), etc.

In studies of propagation of high-frequency wave signals (high frequency, short waves) in layered waveguides it is especially important to take into account the real roughness (nonsmoothness) of surfaces of the waveguide. It is especially important in cases where the length of the wave signal is of the same order with the amplitude or average step of the surface roughness. There is a huge body of references about wave propagation in layered waveguide with perfectly smooth surfaces of attachment of the layers. However, smooth surface is an idealized model for which it is not always possible to rigorously determine or estimate the characteristics of the wave field more accurately, especially in near-surface zones of the waveguide. The roughness of the waveguide layers definitely complicates the mathematical model, but provides opportunity to identify near-surface wave effects and more accurately calculate the quantitative characteristics of the formed wave field in the near-surface area.

There are different theoretical approaches and practical tools for investigating surface waves propagation on rough surfaces (see, for instance, [4-7]). Many papers (see e.g. [8-11]) are dedicated to different cases of normal high-frequency short monochromatic waves stability loses, such as localization of wave energy, internal resonance, occurrence of forbidden zones of frequency, etc.

Possible distortion of the amplitude and phase functions for normal distribution of the wave signal in a weakly rough elastic waveguide are investigated in [12-14]. The occurrence of internal resonance is studied, and conditions for existence of forbidden zones of frequency are revealed using the hypotheses of magneto-electro-elastic layered systems (MELS hypotheses).

In [15], wave propagation in inhomogeneous media with self-similar structure is studied using fractional calculus, along with the space-time discontinuous Galerkin methods. One and two dimensional problems are studied to demonstrate the capability of the proposed model in modeling inhomogeneous media.

In this paper, we propose a new approach for studying the influence of roughness of the surface of the layer-waveguide on the propagation of elastic, normal shear wave by so-called MELS hypotheses.

\section{Problem Statement}

Let us assume that pure shear normal wave signal

$w(x, y, t)=W_{0}(y) \times \exp \left[i\left(k_{0} x-\omega_{0} t\right)\right]$, 
$u(x, y, t) \equiv 0, v(x, y, t) \equiv 0$,

is propagating in elastic, isotropic waveguide $\Omega:=\left\{|x|<\infty ; h_{-}(x) \leq y \leq h_{+}(x) ;|z|<\infty\right\}$ with rough surfaces $y=h_{-}(x)$ and $y=h_{+}(x)$. Here $\omega_{0}$ is the frequency of the source of wave signal, $k_{0} \triangleq\left(2 \pi / \lambda_{0}\right)$ is the wave number and $\lambda_{0}$ is the length of wave signal. Then, the equation of motion of the medium has the following form

$\frac{\partial^{2} w(x, y, t)}{\partial x^{2}}+\frac{\partial^{2} w(x, y, t)}{\partial y^{2}}=c_{0}^{-2} \frac{\partial^{2} w(x, y, t)}{\partial t^{2}}$,

where $c_{0}^{2}=G_{0} / \rho_{0}$ is the speed of the shear normal wave in the waveguide, $G_{0}$ is the shear modulus and $\rho_{0}$ is the density of the waveguide material.

It is assumed, that the roughness of the waveguide surfaces $y=h_{ \pm}(x)$ are represented by the following harmonic functions

$\left\{\begin{array}{l}h_{+}(x)=h_{0}\left[1+\varepsilon_{+} \cdot \sin \left(k_{+} \cdot x\right)+\delta_{+} \cdot \cos \left(k_{+} \cdot x\right)\right], \\ h_{-}(x)=-h_{0}\left[1+\varepsilon_{-} \cdot \sin \left(k_{-} \cdot x\right)+\delta_{-} \cdot \cos \left(k_{-} \cdot x\right)\right],\end{array}\right.$

where $h_{0}$ is the half-thickness of basic layer of the waveguide, $\varepsilon_{ \pm}$and $\delta_{ \pm}$are the relative amplitude coefficients of the heights of roughness profiles with $\left\{\varepsilon_{ \pm} ; \delta_{ \pm}\right\} \ll$, because the heights of the protrusions of roughness $h_{0} \cdot \varepsilon_{ \pm}$and $h_{0} \cdot \delta_{ \pm}$are always much less than the basic layer thickness: $\left\{h_{0} \cdot \varepsilon_{ \pm} ; h_{0} \cdot \delta_{ \pm}\right\} \ll h_{0}, k_{ \pm} \triangleq 2 \pi / \lambda_{ \pm}$is the number of the waviness of roughness profile and $\lambda_{ \pm}$is the step (wavelength) of the roughness profiles.

The boundary conditions on mechanically free non-smooth surfaces of the waveguide $\sigma_{i j}(x, y) \cdot n_{j}^{ \pm}(x)=0$ are written respectively in this form:

$\left.h_{ \pm}^{\prime}(x) \cdot \frac{\partial w(x, y)}{\partial x}\right|_{y=h_{ \pm}(x)}+\left.\frac{\partial w(x, y)}{\partial y}\right|_{y=h_{ \pm}(x)}=0$.

It is evident from (1.3)-(1.5), that its solution must explicitly depend on the roughness of the surfaces. Since the roughness is weak $\left\{h_{0} \cdot \varepsilon_{ \pm} ; h_{0} \cdot \delta_{ \pm}\right\} \ll h_{0}$, the interaction of roughness will mainly be available in case of high-frequency (shortwave) wave signals, for which $\lambda_{0} \sim \lambda_{ \pm} \ll h_{0}$, or equivalently $k_{0} h_{0} \sim k_{ \pm} h_{0} \gg 1$. Then, one might be interested in investigation of the influence of surfaces roughness of the waveguide on the propagation of normal high-frequency shear waves. 


\section{Problem Solution}

There are two methods to solve the problem: the method of successive approximations and the method of introduced hypotheses. Later in this article we will compare wave characteristics of the received wave fields.

\subsection{First Approach}

When high-frequency, normal shear signal (1.1) is propagated in elastic waveguide, interaction of the wave signal with the roughness of the surfaces in the near-surface areas occurs, which consequently leads to amplitude and phase distortion of the primary signal. New harmonics appear and a new amplitude-phase interaction is formed.

We use Fourier method of variables separation, and the solution of the boundary value problem (1.3)-(1.5) is represented in the following form:

$w(x, y, t)=\sum_{n=1}^{\infty} W_{n}(y) \cdot X_{n}(x) \cdot \exp \left(-i \omega_{0 n} t\right)$.

Then the conditions of mechanically free surfaces of the waveguide, on rough surfaces $y=h_{ \pm}(x)$ respectively, for each harmonic of propagating wave will have the following form

$W_{n}^{\prime}\left(h_{ \pm}(x)\right)=\mp h_{0} k_{ \pm} \cdot\left[\varepsilon_{ \pm} \cdot \cos \left(k_{ \pm} \cdot x\right)-\delta_{ \pm} \cdot \sin \left(k_{ \pm} \cdot x\right)\right] \cdot \frac{X_{n}^{\prime}(x)}{X_{n}(x)} W_{n}\left(h_{ \pm}(x)\right)$.

It is suggested, that the equations for determining the desired functions $X_{n}(x)$ and $W_{n}(y)$ are shown in the form

$\left\{\begin{array}{l}W_{n}^{\prime \prime}(y)+k_{n}^{2}\left[\eta_{n}^{2}-1\right] W_{n}(y)=0 \\ X_{n}^{\prime \prime}(x)+k_{n}^{2} X_{n}(x)=0\end{array}\right.$

where the following assignment for appropriate harmonics $\eta_{n}^{2} \triangleq \omega_{n}^{2} k_{n}^{-2} c_{0}^{-2}$ has been taken into account, $k_{n}$ is the wave number (formation coefficient through the thickness of the waveguide), corresponding to the generated $n$-th harmonic.

From surface conditions (2.2) it follows that the undamped solutions of (2.3) in the directions of the propagation $\pm O x$ (for $\operatorname{Im}\left[k_{n}\right] \equiv 0$ ) are shown in the following form

$\left\{\begin{array}{l}W_{n}(y)=C_{1 n} \exp \left(i k_{n} \alpha_{n} y\right)+C_{2 n} \exp \left(-i k_{n} \alpha_{n} y\right) \\ X_{n}(x)=C_{ \pm} \exp \left( \pm i k_{n} x\right)\end{array}\right.$

which, for slow waves, i.e. when $\alpha_{n}^{2} \triangleq \eta_{n}^{2}-1<0$, corresponds to the damped harmonics from the surface up to the depth of the waveguide, and for fast waves, i.e. when $\alpha_{n}^{2} \triangleq \eta_{n}^{2}-1 \geq 0$, corresponds to harmonic forms over the thickness of the waveguide.

From (2.3) it also follows that fast damped waves occur in the directions of wave propagation 
$\pm O x$ in the case of $\operatorname{Re}\left[k_{n}\right] \equiv 0$ :

$\left\{\begin{array}{l}W_{n}^{\prime \prime}(y)-k_{n}^{2}\left[\eta_{n}^{2}-1\right] W_{n}(y)=0 \\ X_{n}^{\prime \prime}(x)-k_{n}^{2} X_{n}(x)=0\end{array}\right.$.

For slow wave, i.e. when $\alpha_{n}^{2} \triangleq \eta_{n}^{2}-1<0$, the solution corresponds to harmonic forms over the thickness of the waveguide, and for fast wave, i.e. when $\alpha_{n}^{2} \triangleq \eta_{n}^{2}-1 \geq 0$, it corresponds to damped harmonics from the surface up to the depth of the waveguide.

Taking into account that the roughness of the surface of the waveguide is weak and its impact on the propagating wave is described by boundary conditions (2.2), the solution of system (2.3) is represented in this form

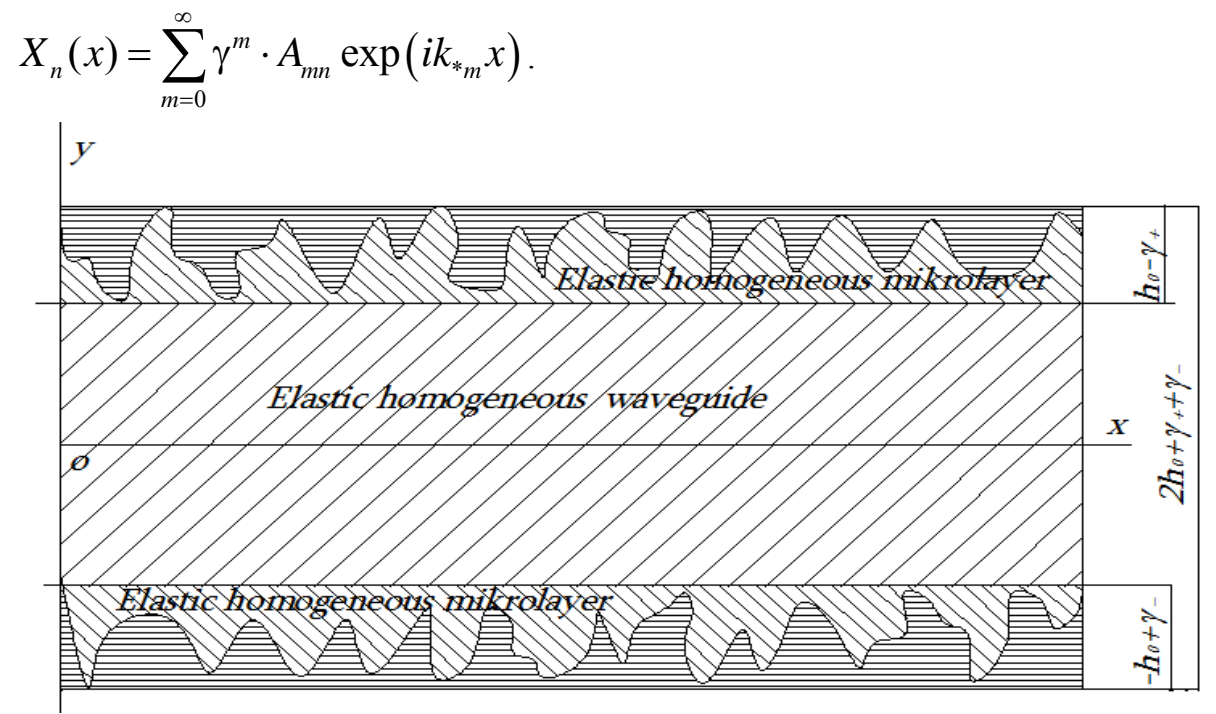

Fig.2.1. The model of elastic waveguide as a multilayer waveguide

Moreover, the value $m=0$ corresponds to the case of homogeneous waveguide. Here, the introduced wave number $k_{*_{m}}$ should be formed by the impact of normal wave signal and roughness of the surfaces of the waveguide.

The roughness of the surfaces, in its turn, is characterized by the greatest common divisor of wave numbers $k_{*} \triangleq \min \left\{k_{+} / p ; k_{-} / q\right\}=2 \pi / \lambda_{*}$ is the smallest common wave number of roughness on the surfaces corresponding to the generated $m_{\text {-th harmonic waves, and }}$ $\gamma \triangleq \max \left\{\sqrt{\varepsilon_{ \pm}^{2}+\delta_{ \pm}^{2}}\right\} \ll 1$ is a small parameter characterizing the weak roughness of the surfaces of the waveguide. 


$$
\begin{aligned}
& k_{n} \alpha_{n}\left[C_{n 1} \exp \left(i k_{n} \alpha_{n} h_{ \pm}(x)\right)-C_{n 2} \exp \left(-i k_{n} \alpha_{n} h_{ \pm}(x)\right)\right] \sum_{m=0}^{\infty} \gamma^{m} \cdot A_{m n} \exp \left(i k_{*_{m}} x\right)= \\
& =\mp h_{0} k_{ \pm} \cdot\left[\varepsilon_{ \pm} \cdot \cos \left(k_{ \pm} \cdot x\right)-\delta_{ \pm} \cdot \sin \left(k_{ \pm} \cdot x\right)\right] \times \\
& \times\left[C_{n 1} \exp \left(i k_{n} \alpha_{n} h_{ \pm}(x)\right)+C_{n 2} \exp \left(-i k_{n} \alpha_{n} h_{ \pm}(x)\right)\right] \sum_{m=0}^{\infty} k_{*_{m}} \gamma^{m} \cdot A_{m n} \exp \left(i k_{*_{m}} x\right)
\end{aligned}
$$

Considering that the right hand sides of boundary conditions (2.6) are in small $m+1$ order in the $n=0$ approximation, for non-trivial solutions of (2.6) we obtain the dispersion equation with the following solution

$\omega_{0 n}=k_{0 n} c_{0}=\frac{2 \pi c_{0}}{\lambda_{0 n}}=\frac{\pi n c_{0}}{h_{0}}$.

Consequently, interaction of the normal wave (1.1) with surface roughness $h_{ \pm}(x)$ is not occur in the $n=0$ approximation, and the propagating wave is still normal as in $n=0$ approximation of longitudinally weakly rough waveguide with mechanically free surfaces [13] $w_{0}(x, y, t)=\sum_{n=1}^{\infty} A_{0 n} \cdot \exp \left[i\left(\frac{\pi n x}{h_{0}}-\omega_{0 n} t\right)\right]$.

From the conditions of synchronization of the surface distortions at the mid-plane of the waveguide $y=0$, we get

$\exp \left[i\left(k_{+m}-k_{-m}\right) x\right]=-\frac{k_{+} \cdot\left[\varepsilon_{+} \cdot \cos \left(k_{+} \cdot x\right)-\delta_{+} \cdot \sin \left(k_{+} \cdot x\right)\right]}{k_{-} \cdot\left[\varepsilon_{-} \cdot \cos \left(k_{-} \cdot x\right)-\delta_{-} \cdot \sin \left(k_{-} \cdot x\right)\right]}$.

Considering that the wave number is formed as $k_{1 n}(x)=k_{+n}-k_{-n}$ and $k_{*} \triangleq \min \left\{p k_{+} ; q k_{-}\right\}=2 \pi / \lambda_{*}$, it is easy to get the allowed wavelengths from (2.9) for the first approximation:

$\lambda_{*}(x)=\lambda_{0} \cdot 2 \pi \arccos ^{-1}\left\{-\frac{k_{+} \cdot\left[\varepsilon_{+} \cdot \cos \left(k_{+} \cdot x\right)-\delta_{+} \cdot \sin \left(k_{+} \cdot x\right)\right]}{k_{-} \cdot\left[\varepsilon_{-} \cdot \cos \left(k_{-} \cdot x\right)-\delta_{-} \cdot \sin \left(k_{-} \cdot x\right)\right]}\right\}$.

Then from the boundary equations (2.6) for the first approximation we will have $\exp \left(i k_{0 n} \alpha_{n}\left(h_{+}(x)-h_{-}(x)\right)\right)-\exp \left(-i k_{0 n} \alpha_{n}\left(h_{+}(x)-h_{-}(x)\right)\right)=0$, therefore formation coefficient of generated distortions of waves is obtained as $k_{0 n} \alpha_{1 n}=\frac{\pi n}{h_{+}(x)-h_{-}(x)}$.

The wave number of the first generated harmonic depends on the surfaces of the non-smooth waveguide 
$k_{1 n}(x)=\left(\frac{\pi n}{h_{0}}\right)\left(h_{+}(x)-h_{-}(x)\right)\left[h_{0}^{2}+\left(h_{+}(x)-h_{-}(x)\right)^{2}\right]^{-1 / 2}$.

In the first approximation, the interaction of the normal wave with surface non-smoothness affects to the propagating wave:

$w_{1}(x, y, t)=\sum_{n=1}^{\infty} W_{1 n}(y) \cdot X_{1 n}(x) \cdot \exp \left(-i \omega_{0 n} t\right)$,

where

$\left\{\begin{array}{l}W_{1 n}(y)=C_{1 n} \exp \left(i \frac{h_{0} \cdot k_{1 n}(x) y}{\left(h_{+}(x)-h_{-}(x)\right)}\right)+C_{2 n} \exp \left(-i \frac{h_{0} \cdot k_{1 n}(x) y}{\left(h_{+}(x)-h_{-}(x)\right)}\right), \\ X_{1 n}(x)=\gamma A_{0 n} \exp \left(i k_{1 n}(x) \cdot x\right) .\end{array}\right.$

Note that if the rough surfaces are "symmetric" with respect to the mid-plane of the waveguide, i.e.

$-h_{-}(x)=h_{+}(x)=h(x)=h_{0}[1+\varepsilon \cdot \sin (k \cdot x)+\delta \cdot \cos (k \cdot x)]$,

then from relations (2.11) and (2.12) for the wave number over the thickness of the waveguide and the coefficient of formation, respectively, are obtained as follows

$k_{1 n}^{s}(x)=\frac{\pi n}{h_{0}} \cdot\left[\frac{h_{0}^{2}}{4 h^{2}(x)}+1\right]^{-1 / 2} ;$

$k_{1 n}^{s}(x) \cdot \alpha_{1 n}^{s}(x)=\frac{\pi n}{h(x)} \cdot\left[\frac{h_{0}^{2}}{h^{2}(x)}+4\right]^{-1 / 2}$.

The solution (2.14) will be correspondingly transformed into

$$
\left\{\begin{array}{l}
W_{1 n}^{s}(y)=C_{1 n}^{s} \exp \left(i\left(\frac{h_{0} \cdot k_{1 n}^{s}(x)}{h(x)}\right) y\right)+C_{2 n}^{s} \exp \left(-i\left(\frac{h_{0} \cdot k_{1 n}^{s}(x)}{h(x)}\right) y\right), \\
X_{1 n}^{s}(x)=\gamma A_{0 n} \exp \left(i k_{1 n}^{s}(x) \cdot x\right) .
\end{array}\right.
$$

In the case of "synchronous" (parallel to each other) roughness on the surfaces of the waveguide, will have the following representations:

$$
\left\{\begin{array}{l}
h_{+}(x)=h_{0}[1+\varepsilon \cdot \sin (k \cdot x)+\delta \cdot \cos (k \cdot x)], \\
h_{-}(x)=-h_{0}[1-\varepsilon \cdot \sin (k \cdot x)-\delta \cdot \cos (k \cdot x)] .
\end{array}\right.
$$

Then from relations (2.11) and (2.12) for the wave number over the thickness of the waveguide and the coefficient of formation, respectively, are obtained as follows:

$$
k_{1 n}^{*}(x)=\frac{\sqrt{5}}{10} \cdot \frac{\pi n}{h_{0}} ; k_{1 n}^{*}(x) \cdot \alpha_{1 n}^{*}(x)=\frac{\sqrt{5}}{20} \cdot \frac{\pi n}{h_{0}} .
$$

The solution (2.14) changes accordingly

$$
34
$$




$$
\left\{\begin{array}{l}
W_{1 n}^{*}(y)=C_{1 n}^{*} \exp \left(i \frac{\sqrt{5}}{20} \cdot \frac{\pi n}{h_{0}} y\right)+C_{2 n}^{*} \exp \left(-i \frac{\sqrt{5}}{20} \cdot \frac{\pi n}{h_{0}} y\right) \\
X_{1 n}(x)=\gamma A_{0 n} \exp \left(i \frac{\sqrt{5}}{10} \cdot \frac{\pi n}{h_{0}} \cdot x\right) .
\end{array}\right.
$$

\subsection{Second Approach}

To analyze the propagation of the normal, pure shear wave signal (1.1) and (1.2), taking into account that in the isotropic waveguide $\Omega:=\left\{|x|<\infty ; h_{-}(x) \leq y \leq h_{+}(x) ;|z|<\infty\right\}$ roughness of surfaces $y=h_{-}(x)$ and $y=h_{+}(x)$ are described by the functions (1.4), the near-surface thin layers with variable thickness (the waveguide is presented as three-layer, see Fig.2) are virtually selected $\Omega=\Omega_{-} \cup \Omega_{0} \cup \Omega_{+}$, where

$$
\left\{\begin{array}{l}
\Omega_{-} \triangleq\left\{|x|<\infty ; h_{-}(x) \leq y \leq-h_{0}+\gamma_{-} ;|z|<\infty\right\}, \\
\Omega_{0} \triangleq\left\{|x|<\infty ;-h_{0}+\gamma_{-} \leq y \leq h_{0}-\gamma_{+} ;|z|<\infty\right\}, \\
\Omega_{+} \triangleq\left\{|x|<\infty ; h_{0}-\gamma_{+} \leq y \leq h_{+}(x) ;|z|<\infty\right\} .
\end{array}\right.
$$

We intend to solve the equation of medium motion (1.3) for all three layers separately with boundary conditions (1.5) on mechanically free, non-smooth surfaces $y=h_{-}(x)$ and $y=h_{+}(x)$ for elastic displacements $w_{ \pm}(x, y, t)$ (respectively for layers $\Omega_{ \pm}$), and the conditions of continuity on virtual cross-sections $y=-h_{0}+\gamma_{-}$and $y=h_{0}-\gamma_{+}$

$$
\begin{aligned}
& \left.w_{0}(x, y, t)\right|_{y=-h_{0}+\gamma_{-}}=\left.w_{-}(x, y, t)\right|_{y=-h_{0}+\gamma_{-}}, \\
& \left.w_{0}(x, y, t)\right|_{y=h_{0}-\gamma_{+}}=\left.w_{+}(x, y, t)\right|_{y=h_{0}-\gamma_{+}}, \\
& \left.\frac{\partial w_{0}(x, y, t)}{\partial y}\right|_{y=-h_{0}+\gamma_{-}}=\left.\frac{\partial w_{-}(x, y, t)}{\partial y}\right|_{y=-h_{0}+\gamma_{-}}, \\
& \left.\frac{\partial w_{0}(x, y, t)}{\partial y}\right|_{y=h_{0}-\gamma_{+}}=\left.\frac{\partial w_{+}(x, y, t)}{\partial y}\right|_{y=h_{0}-\gamma_{+}} .
\end{aligned}
$$

Considering the thinness of the surface layers $\Omega_{ \pm}$, the solution in them are represented with the hypotheses of MELS $[11,13]$ taking into account the nature of the changes arising from surface roughness $y=h_{-}(x)$ and $y=h_{+}(x)$ 


$$
\begin{aligned}
& w_{+}(x, y)=\frac{\operatorname{sh}\left(\mu_{+}\left[y-h_{0}+\gamma_{+}\right]\right)}{\operatorname{sh}\left(\mu_{+}\left[h_{+}(x)-h_{0}+\gamma_{+}\right]\right)} \cdot\left[w_{+}\left(x, h_{+}(x)\right)-w_{0}\left(x, h_{0}-\gamma_{+}\right)\right]+ \\
& +w_{0}\left(x, h_{0}-\gamma_{+}\right) \\
& w_{-}(x, y)=\frac{\operatorname{sh}\left(\mu_{-}\left[y+h_{0}-\gamma_{-}\right]\right)}{\operatorname{sh}\left(\mu_{-}\left[h_{-}(x)+h_{0}-\gamma_{-}\right]\right)} \cdot\left[w_{-}\left(x, h_{-}(x)\right)-w_{0}\left(x,-h_{0}+\gamma_{-}\right)\right]+ \\
& +w_{0}\left(x,-h_{0}+\gamma_{-}\right)
\end{aligned}
$$

where the values $w_{+}\left(x, h_{+}(x)\right)$ and $w_{-}\left(x, h_{-}(x)\right)$ are determined from the conditions on mechanically free surface (1.5) as follows:

$$
\begin{aligned}
& w_{+}\left(x, h_{+}(x)\right)=\frac{\mu_{+} \cdot \operatorname{cth}\left(\mu_{+}\left[h_{+}(x)-h_{0}+\gamma_{+}\right]\right) \cdot\left[1-\left\{h_{+}^{\prime}(x)\right\}^{2}\right]}{\mu_{+} \cdot \operatorname{cth}\left(\mu_{+}\left[h_{+}(x)-h_{0}+\gamma_{+}\right]\right) \cdot\left[1-\left\{h_{+}^{\prime}(x)\right\}^{2}\right]+h_{+}^{\prime}(x)} \cdot w_{0}\left(x, h_{0}-\gamma_{+}\right) ; \\
& w_{-}\left(x, h_{-}(x)\right)=\frac{\mu_{-} \cdot \operatorname{cth}\left(\mu_{-}\left[h_{-}(x)+h_{0}-\gamma_{-}\right]\right) \cdot\left[1-\left(h_{-}^{\prime}(x)\right)^{2}\right]}{\mu_{-} \cdot \operatorname{cth}\left(\mu_{-}\left[h_{-}(x)+h_{0}-\gamma_{-}\right]\right) \cdot\left[1-\left(h_{-}^{\prime}(x)\right)^{2}\right]+h_{-}^{\prime}(x)} \cdot w_{0}\left(x,-h_{0}+\gamma_{-}\right) .
\end{aligned}
$$

Substituting (2.27) and (2.28) into (2.25) and (2.26), we reach the solution in the near-surface thin layers of the waveguide formed by the propagation of the normal wave $w_{0}(x, y, t)=W_{0}(y) \cdot \exp \left[i\left(k_{0} x-\omega_{0} t\right)\right]$ in the basic layer $\Omega_{0}$ :

$$
\begin{aligned}
& w_{+}(x, y)=\left\{\begin{array}{l}
1 \frac{\operatorname{sh}\left(\mu_{+}\left[y-h_{0}+\gamma_{+}\right]\right)}{\operatorname{sh}\left(\mu_{+}\left[h_{+}(x)-h_{0}+\gamma_{+}\right]\right)} \times \\
\times \frac{h_{+}^{\prime}(x)}{\mu_{+} \cdot \operatorname{cth}\left(\mu_{+}\left[h_{+}(x)-h_{0}+\gamma_{+}\right]\right) \cdot\left[1-\left\{h_{+}^{\prime}(x)\right\}^{2}\right]+h_{+}^{\prime}(x)}
\end{array}\right\} \cdot w_{0}\left(x, h_{0}-\gamma_{+}\right) ; \\
& w_{-}(x, y)=\left\{\begin{array}{l}
1-\frac{\operatorname{sh}\left(\mu_{-}\left[y+h_{0}-\gamma_{-}\right]\right)}{\operatorname{sh}\left(\mu_{-}\left[h_{-}(x)+h_{0}-\gamma_{-}\right]\right)} \times \\
\left.x \frac{h_{-}^{\prime}(x)}{\mu_{-} \cdot \operatorname{cth}\left(\mu_{-}\left[h_{-}(x)+h_{0}-\gamma_{-}\right]\right) \cdot\left[1-\left(h_{-}^{\prime}(x)\right)^{2}\right]+h_{-}^{\prime}(x)}\right\} \cdot w_{0}\left(x,-h_{0}+\gamma_{-}\right) .
\end{array}\right.
\end{aligned}
$$

Let us represent the normal wave in the basic layer $\Omega_{0}$ in a common form

$$
w_{0}(x, y, t)=\left[A \cos \left(\mu_{*} y\right)+B \sin \left(\mu_{*} y\right)\right] \cdot \exp \left[i\left(k_{*} x-\omega_{0} t\right)\right] \text {, }
$$


here $k_{*} \triangleq \min \left\{p k_{+} ; q k_{-}\right\}=2 \pi / \lambda_{*}$ is the smallest common wave number of the roughness on the surfaces corresponding to the generated harmonic of the wave.

From the conditions of continuity of mechanical stresses (2.24), we obtain a dispersion equation to determine the formation coefficient $\mu_{*}$ :

$$
\begin{aligned}
& \mu_{*}^{2}-\mu_{*} \cdot \operatorname{ctg}\left(\mu_{*}\left(2 h_{0}-\left(\gamma_{+}+\gamma_{-}\right)\right) \cdot\left(f_{+}\left(\mu_{+} ; h_{+}(x)\right)-f_{-}\left(\mu_{-} ; h_{-}(x)\right)\right)=\right. \\
& =-f_{+}\left(\mu_{+} ; h_{+}(x)\right) \cdot f_{-}\left(\mu_{-} ; h_{-}(x)\right),
\end{aligned}
$$

in which

$$
\begin{aligned}
& f_{+}\left(\mu_{+} ; h_{+}(x)\right) \triangleq\left[\begin{array}{l}
\frac{1}{\operatorname{sh}\left(\mu_{+}\left[h_{+}(x)-h_{0}+\gamma_{+}\right]\right)} \times \\
\left.\times \frac{1}{\mu_{+} \cdot \operatorname{cth}\left(\mu_{+}\left[h_{+}(x)-h_{0}+\gamma_{+}\right]\right) \cdot\left[1-\left\{h_{+}^{\prime}(x)\right\}^{2}\right]+h_{+}^{\prime}(x)} \cdot \mu_{+} h_{+}^{\prime}(x)\right],
\end{array}\right] \\
& f_{-}\left(\mu_{-} ; h_{-}(x)\right) \triangleq\left[\begin{array}{l}
\frac{1}{\operatorname{sh}\left(\mu_{-}\left[h_{-}(x)+h_{0}-\gamma_{-}\right]\right)} \times \\
\times \frac{1}{\mu_{-} \cdot \operatorname{cth}\left(\mu_{-}\left[h_{-}(x)+h_{0}-\gamma_{-}\right]\right) \cdot\left[1-\left\{h_{-}^{\prime}(x)\right\}^{2}\right]+h_{-}^{\prime}(x)} \cdot \mu_{-} h_{-}^{\prime}(x)
\end{array}\right] .
\end{aligned}
$$

They characterize the influence of the rough surfaces on the formation coefficient.

It is obvious, that the solution of the dispersion equation (2.32) significantly depends on the surface roughness $h_{ \pm}(x)$.

\section{Numerical Analysis of Obtained Results}

Considering the surface roughness, in the first approach, the solutions for formation coefficient $k_{0 n} \alpha_{1 n}$ and wave number $k_{1 n}(x)$ are obtained in the forms (2.11) and (2.12) respectively. As expected, the variable thickness through the waveguide plays the main role in these expressions $\xi(x) \triangleq h_{+}(x)-h_{-}(x)$, by means of which the wave process can be controlled.
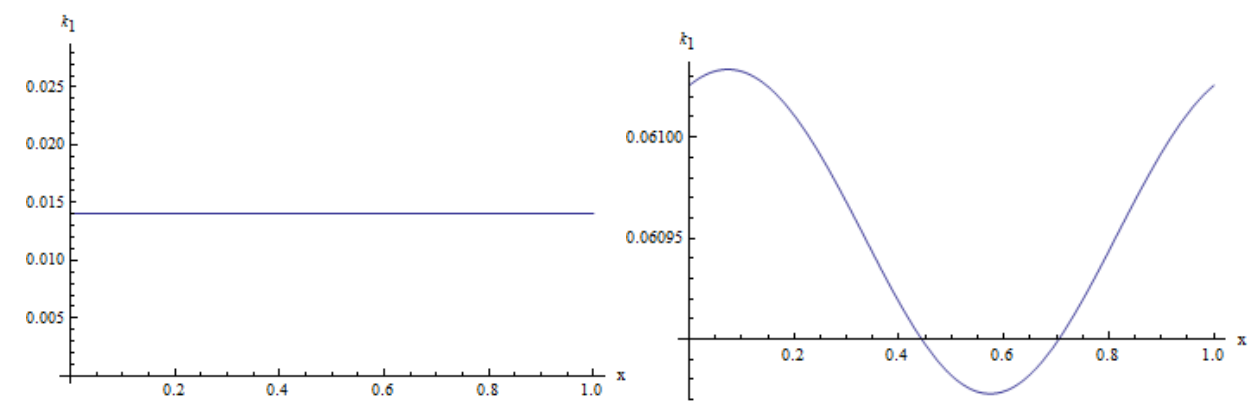

Fig. 3.1. The wave number for "synchronous" and "symmetric" surface roughness of the waveguide (the first approach) 
Graphics of the formation coefficient and the wave number for different particular characteristic surfaces of roughness are given in Figs. 3.1 and 3.2 using the relations (2.15)(2.21), respectively. From the figures of the wave number and formation coefficient it follows that for "symmetric" surface roughness of the waveguide (2.15) the changes of these values are characteristically different from the case of "synchronous" surface roughness (2.19).
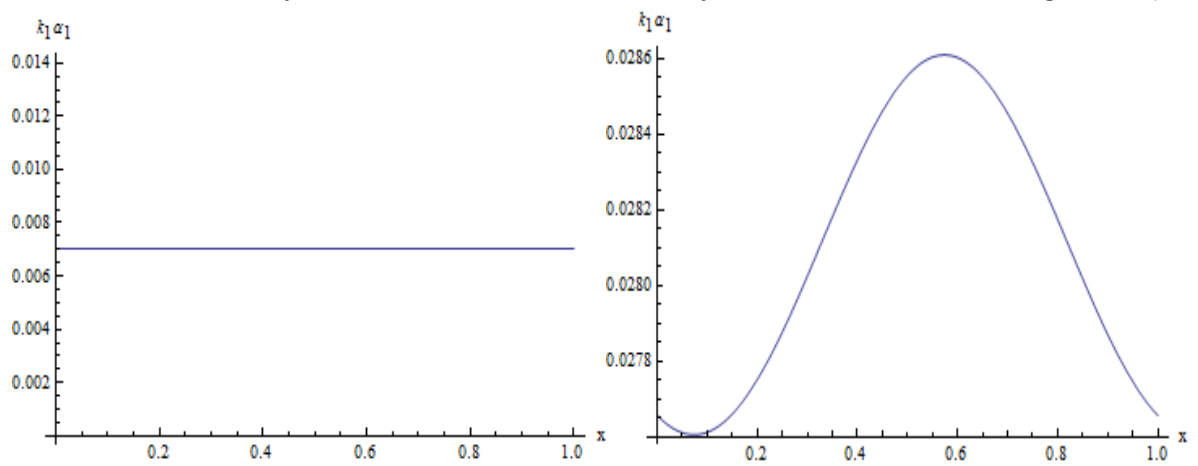

Fig. 3.2. The formation coefficient for "synchronous" and "symmetric" surface roughness of the waveguide (the first approach)

From Figs. 3.1 and 3.2 it is obvious that in the case of "symmetric" surface roughness of the waveguide (2.15), the wave number and the formation coefficient are periodically changed with respect to the half-thickness of the waveguide in the interval $x \in\left[0 ; \lambda_{*}\right]$.

In the case of "synchronous" surface roughness of the waveguide (2.19) the wave number and the formation coefficient are only changed by a constant value for each $n$-th harmonic. In the general case of arbitrary surface roughness $y=h_{-}(x)$ and $y=h_{+}(x)$ from (2.9)(2.14) it follows that due to the difference of surface roughness in the near-surface areas there occur qualitatively identical, but quantitatively different harmonics, a synchronization which occurs at the mid-plane $y=0$. From (2.11) and (2.12) it is obvious that the wave number $k_{1 n}(x)$ and the formation coefficient $k_{0 n} \alpha_{1 n}(x)$ for the propagation of the waves is always positive, since $h_{+}(x)-h_{-}(x)>0$. From relations (2.18) and (2.21) we can easily get the nature of the changes of elastic shear through the thickness of the waveguide, according to the variable thickness of the waveguide (see Figs. 3.3 and 3.4). The picture of elastic shear $W_{1 n}^{s}(y)$ over the thickness of the waveguide for the "symmetric" surface roughness is defined by relation (2.18) and is shown in Fig.3.3. Fig. 3.3 shows that over the thickness of the waveguide for the "symmetric" surface roughness (2.15), the normal waveform is periodically distorted depending on the law of variation of its thickness $\xi(x) \triangleq h_{+}(x)-h_{-}(x)$. Accordingly, the phase velocity of the generated harmonic is also changed. The elastic shear $W_{1 n}^{*}(y)$ over the thickness of the waveguide for "synchronous" surface roughness is defined by relation (2.21) and is shown in Fig.3.4. From (2.20) it follows 
that in this case only short waves with lengths $\lambda_{*}=\sqrt{5} \cdot h_{0} / n$ propagate for large numbers of harmonics $n$, such that $n \lambda_{*} \ll \sqrt{5} \cdot h_{0}$.

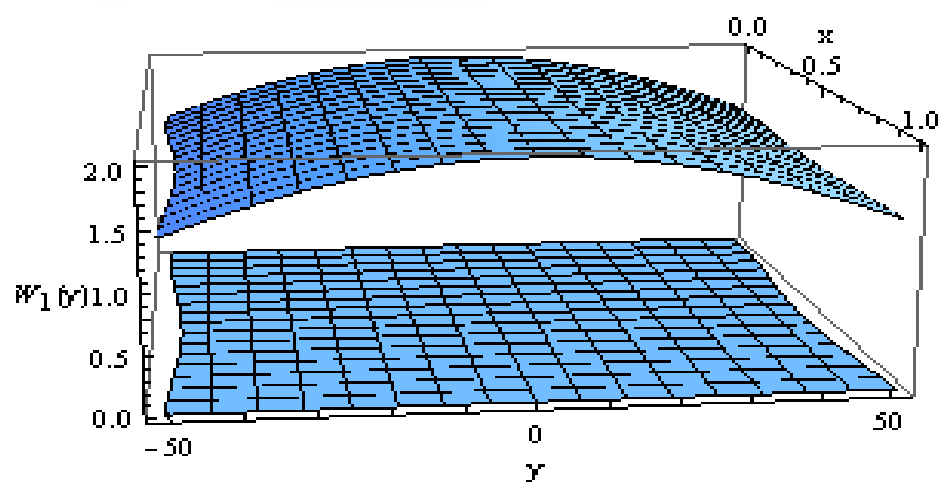

Fig. 3.3. The elastic shear through the thickness of the waveguide for "symmetric" surface roughness (the first approach)

Solving the problem with the method of hypotheses MELS, through the thickness of the waveguide we obtain the expression of elastic shear in the basic layer $\Omega_{0}$ in the form of (2.31), which is analytically continued in both near-surface zones $\Omega_{-}$and $\Omega_{+}$, accordingly (2.30) and (2.29). The image over the thickness of the waveguide is constructed after determining the formation coefficient $\mu_{*}$ from the dispersion equation (2.32). From relations (2.29)-(2.34) it is obvious that the solutions, received in the near-surface zones $\Omega_{-}$and $\Omega_{+}$, are characteristically the same, but numerically different at different surface roughness $h_{+}(x)$ and $h_{-}(x)$.

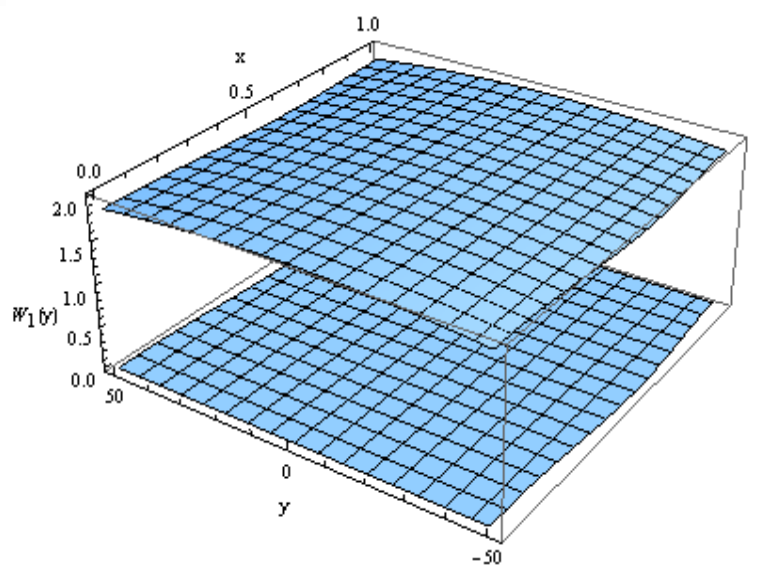

Fig. 3.4. The elastic shear through the thickness of the waveguide for "synchronous" surface roughness (the first approach) 
The dispersion equation (2.32) is much simplified in the cases of "symmetric" (2.15) and "synchronous"

(2.19) surface roughness, considering the expressions of the coefficients of the dispersion equation $f_{+}\left(\mu_{+} ; h_{+}(x)\right)$ and $f_{-}\left(\mu_{-} ; h_{-}(x)\right)$, in relations (2.33) and (2.34) respectively.

Fig. 3.5 shows the graphical dependence of the formation coefficient $\mu_{*}$ on $X$.
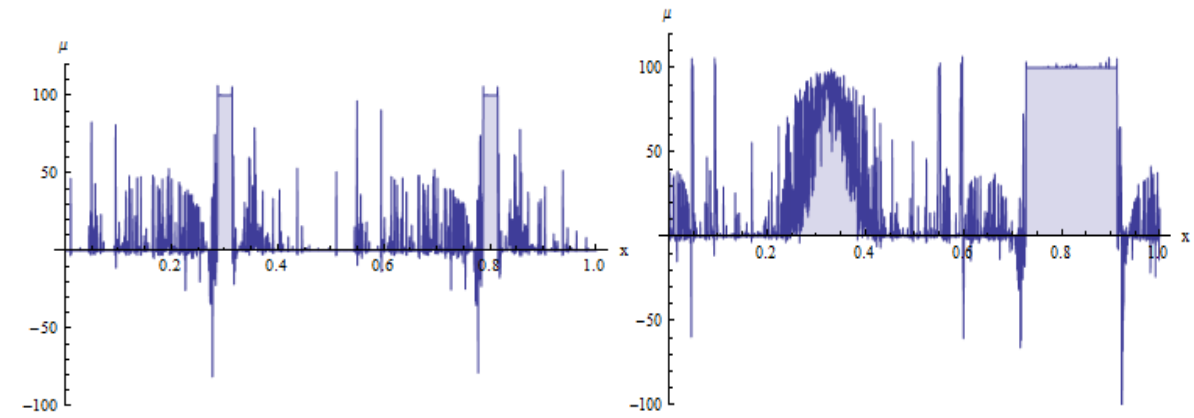

Fig. 3.5. The formation coefficient for "synchronous" and "symmetric" surface roughness of the waveguide (the second approach)

To each formation coefficient $\mu_{*_{\mathrm{n}}}$ naturally corresponds a wave number $k_{*_{n}}=2 \pi / \lambda_{*_{n}}=\sqrt{\omega_{0 n}^{2} C_{0}^{-2}-\mu_{*_{n}}^{2}}$.

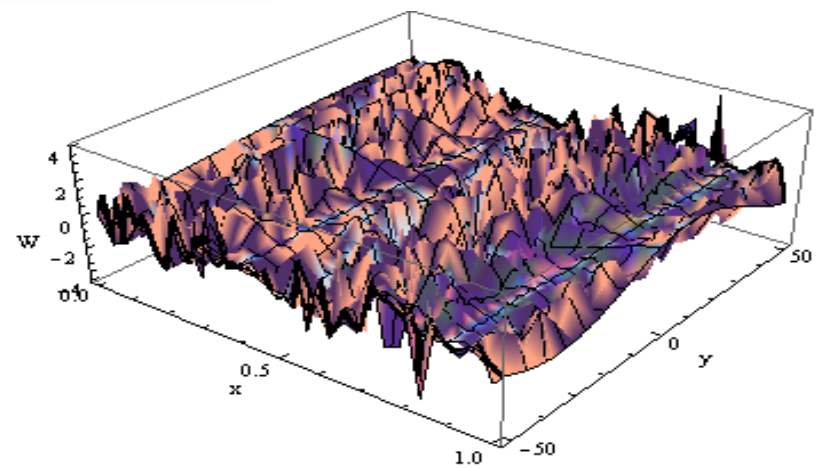

Fig. 3.6. The elastic shear through the thickness of the waveguide for "symmetric" surface roughness (the second approach)

From the dispersion equation (2.32) and the relations (2.33) and (2.34) it is evident that in the absence of roughness on the surfaces of the waveguide, i.e. when $h_{+}^{\prime}(x)=h_{-}^{\prime}(x) \equiv 0$, both introduced multipliers (2.33) and (2.34) become zero and from the dispersion equation we obtain the case of homogeneous waveguide $\mu_{*_{n}}=\mu_{0 n}=\pi n / h_{0}$.

From the obtained graphs it is also seen how the presence of "symmetric" (2.15) or "synchronous" (2.19) surface roughness of relatively homogeneous waveguide leads to distortion of forms (formation coefficient $\mu_{*_{n}}$ and wave number $k_{*_{n}}$ ). 


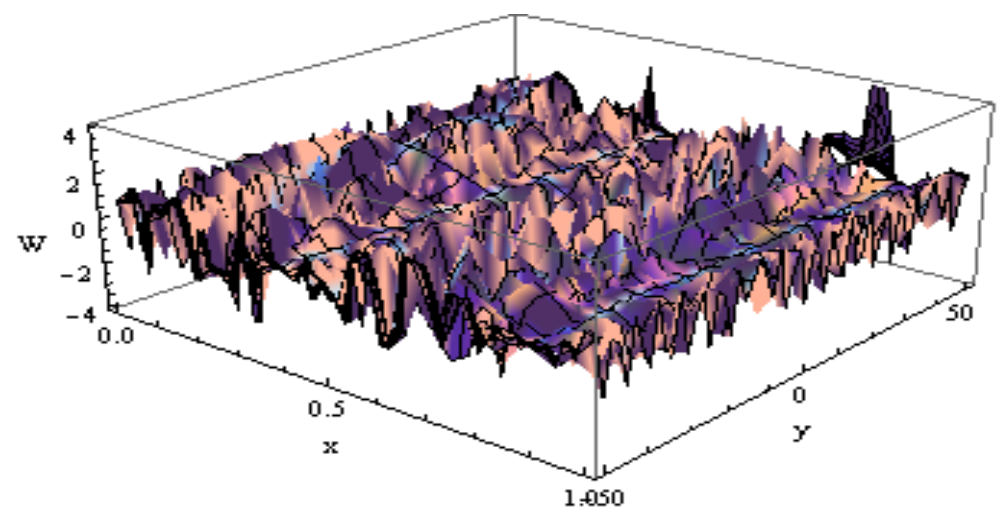

Fig. 3.7. The elastic shear through the thickness of the waveguide for "synchronous" surface roughness (the second approach)

From relations (2.32)-(2.34) and the received graphs it is also clear that weak surface roughness do not lead to appearance of damped propagating harmonics through the depth of the waveguide. Partial localization of the wave energy occurs only in the thin surface rough layers, which can be seen in the given figures of elastic shear over the thickness of the waveguide. The images of elastic shear throughout the thickness of the waveguide in particular "symmetric" (2.15) and "synchronous" (2.19) surface roughness cases are shown in Figs. 3.6 and 3.7.

\section{Conclusion}

It is shown that weak surface roughness lead to instability of a normal propagating wave in the waveguide. The presence of surface roughness can lead to prohibition of waves of certain lengths depending on the characteristic values of the functions of the roughness. Only partial localization of wave energy in thin near-surface areas of roughness occurs. The localized surface waves do not occur. The introduced method of hypotheses MELS allows to analyze the process of distortion of the normal waves, that will make it convenient for studies of wave processes in waveguides with complicated properties and sophisticated characteristic roughness of the material of the waveguide and its surfaces.

\section{References}

1. Potel C., Bruneua M., N'Djomo L.C.F., Leduc D., Elkettani M.E., Izbicki J.-L. Shear horizontal acoustic waves propagating along two isotropic solid plates bonded with a nondissipative adhesive layer: Effects of the rough interfaces. //Jour. Of Appl. Physics, vol.118, (2015).

2. Valier-Brasier T., Potel C., Bruneau M., Leduc D., Morvan B., Izbicki J.-L. Coupling of shear acoustic waves by gratings: Analytical and experimental analysis of spatial periodicity effects. Acta Acust 97(5), 717-727 (2011).

3. Banerjee S., Kundu T. Elastic wave propagation in sinusoidally corrugated waveguides. //J. Acoust. Soc. Am.119 (4), 2006-2017 (2006).

4. Biryukov S.V., Gulyaev Y.V., Krylov V., Plessky V. Surface acoustic waves in inhomogeneous media. Springer Series on Wave Phenomena. Vol. 20, 388 (1995).

5. Brekhovskikh L. Waves in Layered Media, 2nd ed. Applied Mathematics and Mechanics, Elsevier Science. Vol. 16, 520 (2012) 
6. Royer D., Dieulesaint E. Elastic Waves in Solids I: Free and Guided Propagation. Springer Science \& Business Media, 374 (2000).

7. Apostol F.B. The Effect of Surface Inhomogeneities on the Propagation of Elastic Waves. //Journal of Elasticity. Vol. 114, Issue 2, 85-99 (2014).

8. Golub M.V., Zhang C. In-plane time-harmonic elastic wave motion and resonance phenomena in a layered phononic crystal with periodic cracks. //J. Acoust. Soc. Am. Vol.137, Issues 1, 238 (2015)

9. Piliposyan D.G., Ghazaryan K.B., Piliposyan G.T. Internal resonances in a periodic magneto-electro-elastic structure. //J. Appl. Phys., vol. 116, 044107 (2014)

10. Vashishth A.K., Vishakha Gupta. Wave propagation in transversely isotropic porous piezoelectric materials: //Int. J. of Solids and Structures, vol. 46, 3620-3632 (2009).

11. Triantafyllidis N., Elias C. Aifantis. A gradient approach to localization of deformation. I. Hyperelastic materials. //Journal of Elasticity. Vol. 16, Issue 3, 225-237 (1986).

12. Avetisyan A.S. On the formulation of the electro-elasticity theory boundary value problems for electro-magneto-elastic composites with interface roughness. //Proc. of NAS Armenia, ser. Mechanics, vol. 68, №2, 29-42 (2015).

13. Hunanyan A.A. The instability of shear normal wave in elastic waveguide of weakly inhomogeneous material. //Proc. of NAS Armenia, ser. Mechanics, vol. 69, №3, 29-40 (2016)

14. Avetisyan A.S., Hunanyan A.A. The efficiency of application of virtual cross-sections method and hypotheses MELS in problems of wave signal propagation in elastic waveguides with rough surfaces. //Journal of Advances in Physics, vol. 11, №7, 3564 3574 (2016)

15. Hüseyin Gökmen Aksoy. Wave Propagation in Heterogeneous Media with Local and Nonlocal Material Behavior. //Journal of Elasticity. Vol. 122, Issue 1, 1-25 (2016).

Funding: This study was funded by RA MES State Committee of Science (grant number 15T-2C026).

\section{About authors:}

A.S. Avetisyan - Corresponding Member of NAS, Professor, Institute of Mechanics, National Academy of Sciences, Yerevan, Republic of Armenia

Address: 0019, Yerevan, ave. Baghramyan, 24/2

Phone: (+37493) 00-44-55, E-mail: ara.serg.avetisyan@gmail.com

A.A. Hunanyan - PhD student, Institute of Mechanics, National Academy of Sciences, Yerevan, Republic of Armenia

Address: 0019, Yerevan, ave. Baghramyan, 24/2

Phone: (+37491) 77-55-33, E-mail: hunanyan.areg21@gmail.com

Received 12.04.2017 